\title{
Evaluation of multi-component interventions for prevention of nosocomial pneumonia in older adults: a randomized, controlled trial
}

\author{
Barbara H. Rosario ${ }^{1}$ (D) Humaira Shafi ${ }^{2}$ - Anthony C. A. Yii ${ }^{3}$. Louis Y. Tee ${ }^{1}$. Arron S. H. Ang ${ }^{4} \cdot$ Gek Kheng Png $^{1}$. \\ Wendy S. T. Ang ${ }^{1} \cdot$ Yan Qing Lee ${ }^{1} \cdot$ Pei Ting Tan ${ }^{5} \cdot$ Aniruddha Sahu ${ }^{1} \cdot$ Lin Fang Zhou ${ }^{1} \cdot$ Yi Ling Zheng ${ }^{1}$. \\ Roslinda Binte Slamat ${ }^{1} \cdot$ Aza A. M. Taha ${ }^{3}$
}

Received: 2 November 2020 / Accepted: 28 April 2021 / Published online: 3 June 2021

(c) European Geriatric Medicine Society 2021

\section{Key Summary points}

Aim RCT to evaluate the efficacy of multi-component interventions for prevention of hospital-acquired pneumonia in hospitalized older patients.

Findings The multi-component interventions did not reduce hospital-acquired pneumonia but increased the mean time to next hospitalisation due to respiratory infection (11.5 months vs. 9.5 months; $P=0.049$ ), and reduced the risk of hospitalisation in 1 year $(18.6 \%$ vs. $34.4 \% ; P=0.049)$. This was likely due to the increased recognition of oropharyngeal dysphagia (35.6\% vs. 20.3\%; $P<0.001)$ and improved influenza $(54.5 \%$ vs $17.2 \% ; P<0.001)$ and pneumococcal vaccination rates (52.5\% vs. $20.3 \% ; P<0.001)$.

Message A multi-component intervention for nosocomial pneumonia may not significantly reduce the incidence of hospitalacquired pneumonia but significantly increases the frequency of diagnosis of oropharyngeal dysphagia, improves vaccination rates and can reduce future hospitalisations for respiratory infections in older adults.

\begin{abstract}
Aims To evaluate the efficacy of multi-component interventions for prevention of hospital-acquired pneumonia in older patients hospitalized in geriatric wards.

Methods A randomized, parallel-group, controlled trial was undertaken in patients aged 65 and above who were admitted to a tertiary hospital geriatric unit from January 1, 2016 to June 30, 2018 for an acute non-respiratory illness. Participants were randomized by to receive either a multi-component intervention (consisting of reverse Trendelenburg position, dysphagia screening, oral care and vaccinations), or usual care. The outcome measures were the proportion of patients who developed hospital-acquired pneumonia during hospitalisation, and mean time from randomization to the next hospitalisation due to respiratory infections in 1 year.
\end{abstract}

Barbara H. Rosario

rosario.barbara.helen@singhealth.com.sg

1 Department of Geriatric Medicine, Changi General Hospital, 2 Simei Street 3, Singapore 529889, Singapore

2 Department of Infectious Diseases, Changi General Hospital, Singapore, Singapore

3 Department of Respiratory and Critical Care Medicine, Changi General Hospital, Singapore, Singapore

4 Department of Accident and Emergency, Changi General Hospital, Singapore, Singapore

5 Clinical Trials and Research Unit, Changi General Hospital, Singapore, Singapore 
Results A total of 123 participants (median age, $85 ; 43.1 \%$ male) were randomized, $(n=59)$ to intervention group and $(n=64)$ to control group. The multi-component interventions did not significantly reduce the incidence of hospital-acquired pneumonia but did increase the mean time to next hospitalisation due to respiratory infection (11.5 months vs. 9.5 months; $P=0.049$ ), and reduced the risk of hospitalisation in 1 year (18.6\% vs. $34.4 \% ; P=0.049)$. Implementation of multi-component interventions increased diagnoses of oropharyngeal dysphagia $(35.6 \%$ vs. $20.3 \% ; P<0.001)$ and improved the influenza $(54.5 \%$ vs $17.2 \% ; P<0.001)$ and pneumococcal vaccination rates $(52.5 \%$ vs. $20.3 \% ; P<0.001)$.

Conclusions The nosocomial pneumonia multi-component intervention did not significantly reduce the incidence of hospitalacquired pneumonia during hospitalisation but reduce subsequent hospitalisations for respiratory infections.

Clinical Trial Registration ClinicalTrial.gov, NCT04347395.

Keywords Nosocomial infection $\cdot$ Pneumonia $\cdot$ Older adults $\cdot$ Multi-component interventions $\cdot$ Randomized controlled trial

\section{Introduction}

Among all the hospital-acquired infections, nosocomial pneumonia has the highest incidence and highest mortality [1-3]. Five to 21 per 1000 hospital admissions develop hospital-acquired pneumonia, which has an attributable mortality rate of $33-50 \%[1,2]$. In intensive care units, multicomponent interventions, consisting of semi-recumbent positioning at $30^{\circ}-45^{\circ}$, gastric acid suppression and oral care, have been shown to effectively reduce the incidence of ventilator-associated pneumonia [4-7]. Yet it is unclear if these results can be extrapolated to the older population in the general geriatric wards. To investigate whether these interventions may also benefit older adults in the general geriatric wards, we designed a multi-component intervention for hospital-acquired pneumonia that aims to mitigate the risk factors that are more prevalent in older adults.

The older population is more susceptible to hospitalacquired pneumonia, and suffers from more severe infections and worse outcomes [8,9]. Older adults are more vulnerable due to multiple factors, such as weaker immune responses and higher risk of oropharyngeal dysphagia as a result of age-related changes, frailty and other co-morbidities [10-15]. Older adults have poorer oral hygiene and inadequate oral care during hospitalisation can contribute towards oral and gastrointestinal colonization by multi-drug resistant organisms and an increased risk of aspiration pneumonia [16-19].

Locally the influenza-associated hospitalisation rate is $16.3 \%$ with substantially increased excess hospitalisation rates in those age 75 years and above [20]. Although guidelines recommend routine vaccination of older adults, vaccination uptake rates are low, with vaccination rates of only $17 \%$ for influenza and $6.1 \%$ for pneumococcus among older adults in Singapore [21-23].

We hypothesized that a multi-component intervention will reduce the incidence of hospital-acquired pneumonia in older adults, and prevent future hospitalisations for respiratory infections. The multi-component intervention comprised of four components: reverse Trendelenburg, dysphagia screen, oral care and vaccinations. To test this hypothesis, we performed a randomized, parallel-arm, controlled trial with one arm receiving the multi-component intervention and the other arm receiving usual care. The incidence of hospital-acquired pneumonia during index hospitalisation, as well as the time from randomization to readmission to hospital for respiratory infection in 1 year, was compared between both arms.

\section{Methods}

\section{Study design and oversight}

This randomized, parallel-group, controlled trial was performed from January 1, 2016 to June 30, 2018 in the geriatric unit at Changi General Hospital, a tertiary care hospital in Singapore. The trial was conducted in accordance with the Declaration of Helsinki, and the study protocol was approved by the SingHealth Centralized Institutional Review Board. Eligible patients were aged 65 years and older and admitted via the Accident \& Emergency department for an acute non-respiratory illness with an anticipated hospital length of stay (LOS) greater than $72 \mathrm{~h}$. Patients admitted with acute spinal cord injury or full-thickness/unstageable pressure injuries were excluded because the prolonged reverse Trendelenburg position may be contraindicated. To ensure hospital acquired pneumonia was not already present, patients who had been hospitalized for more than $72 \mathrm{~h}$ before recruitment were excluded. Written informed consent was obtained by a study team member and if the patient lacked capacity to make decisions then consent was obtained from a legally appointed representative (LAR).

Randomization was performed by distributing sealed, opaque envelopes with a 1:1 allocation to two arms. Block randomization was performed. Patient was randomized in a $1: 1$ ratio with block size of 10 . One arm received the multicomponent intervention, while the other arm received usual 
care. Blinding was not possible since the intervention group received perceptibly different care, such as reverse Trendelenburg, compared to the control group.

The multi-component intervention that we developed consisted of four components. The first and novel aspect of the study was that we placed the bed in a reverse Trendelenburg position (Fig. 1) with the whole bed tilted to approximately $30^{\circ}$ [24] to reduce aspiration of respiratory pathogens and gastric contents throughout the hospitalisation. Second, we performed routine screening for oropharyngeal dysphagia at randomisation to the study, which was within $72 \mathrm{~h}$ of hospital admission, to lower the risk of aspiration when eating. Screening for oropharyngeal dysphagia was undertaken by the research team members who were trained by a certified speech therapist (ST). The dysphagia screening test was adapted from the Toronto Bedside Swallowing Screening Test (TOR_BSST) [25]. Screening components included bedside observations (alertness, ease of breathing, facial and oromotor functions) as well as a swallowing assessment. Patients who did not meet the components on the screening criteria were deemed to have "failed" the screening and were referred to a certified ST for evaluation. The dysphagia screen is shown in Fig. 4. Third, we prescribed oral chlorhexidine to diminish the bacterial colonization of the oropharynx and gastrointestinal tract. Chlorhexidine was chosen because it has been shown to reduce the risk of ventilator-associated pneumonia [26, 27]. Oral care initially comprised of chlorhexidine $0.2 \%$ gargle served four times a day (in addition to the patient's usual oral care such as tooth brushing or denture cleansing). If the patient was unable to use gargle then oral swabbing would be undertaken but the frequency was reduced to twice a day on August 1, 2017 due to inadequate adherence by patients. Fourth, we offered appropriate vaccinations for influenza and pneumococcus, to boost immunity against respiratory

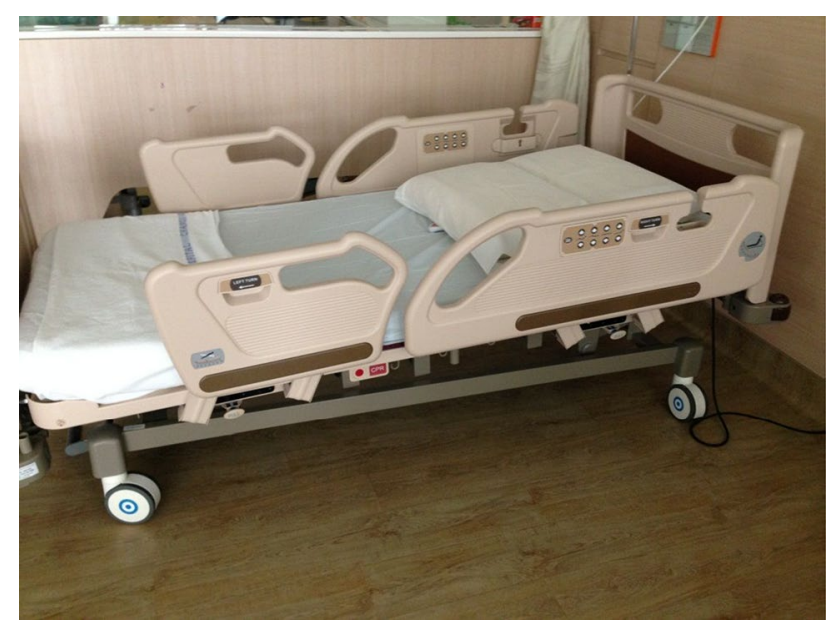

Fig. 1 Reverse Trendelenburg position or whole bed tilt infections either prior to hospital discharge or administered 2-6 weeks after discharge in those with an episode of sepsis during their hospital admission. On discharge, patients were advised to prop their heads up on pillows when sleeping, and to avoid lying flat for an hour after meals, to reduce the risk of gastric aspiration.

Usual care did not impose any recommendation on the patient's position during their hospital admission. Speech therapists would only assess patients for oropharyngeal dysphagia during the index hospital admission if dysphagia was clinically suspected by the medical team. Standard oral care involved using a swab soaked in diluted sodium bicarbonate solution to clean the oral cavity whenever the patient desired (in addition to the patients usual oral care such as tooth brushing or denture cleansing), and vaccinations were not discussed routinely with patients on discharge unless requested by the patient or their family.

The primary outcome was the incidence of hospitalacquired pneumonia during hospitalisation, and the secondary outcome was the time from randomization to a subsequent hospitalisation due to an acute respiratory infection. Hospital acquired pneumonia was defined as any pneumonia that developed more than $48 \mathrm{~h}$ after an acute hospital admission with two or more of the following; positive chest imaging, clinical signs and symptoms, laboratory evidence [28, 29]. Respiratory infections from all causes, upper respiratory tract infection (URTI), lower respiratory tract infection (LRTI) and pneumonia (community acquired, aspiration, bacterial or viral) that resulted in a hospital admission were included in the re-admission analysis. The study team called participants 90 days and 1 year after discharge, to ascertain any hospitalisations with respiratory infections or hospital admissions from other causes, and reviewed electronic medical records up to 1 year after discharge to determine any subsequent hospitalisation due to respiratory infection or mortality. Outcome measures included the frequency of diagnosis of oropharyngeal dysphagia and vaccination rates. Study team members reviewing outcomes were not blinded.

\section{Statistical analysis}

We assumed that the multi-component interventions would reduce the incidence of hospital-acquired pneumonia during hospitalisation by $50 \%$ compared to usual care (10\% vs. $20 \%$ ), with $90 \%$ power at a two-sided significance level of $5 \%$. The power calculation was based on an audit of pneumonia undertaken at the geriatric unit. This calculation resulted in a sample size of 540 participants. Analysis of the primary outcomes included all participants who received study interventions. For the proportion of participants who developed hospital-acquired pneumonia during hospitalisation, the two arms were compared using a Chi-squared test. The time to the next hospitalisation due to respiratory 
infection was modelled using Kaplan-Meier analysis with a log-rank test. For the survival analysis, patients were censored if they died before occurrence of respiratory infection, or 1 year had elapsed if respiratory infection did not occur. We did not censor patients if they were readmitted for reasons other than respiratory infection.

Baseline characteristics and secondary outcomes were analyzed based on their statistical distributions. Continuous data with parametric distributions were compared using two-tailed $t$ tests, while non-parametric distributions were compared using Mann-Whitney $U$ tests. The difference between participant characteristics and assigned group was performed using Chi-square test or Fishers Exact Test for categorical data. Proportions were compared using Chisquared tests. Statistical analysis was performed using SPSS statistical software, version 20.0.

\section{Results}

\section{Baseline characteristics}

Recruitment took place between January 1, 2016 and June 30, 2018 (Fig. 2). Of the 1500 patients screened, 124 were eligible and gave written informed consent. One patient withdrew consent before study interventions commenced and 123 patients are included in the analysis. 59 patients were assigned to the intervention group and 64 were assigned to the control group. Six patients (three in each arm) were lost to follow-up by telephone but outcome measures were obtained from the electronic medical records. The study was terminated before reaching the calculated sample size of 540 due to slow recruitment.

The baseline characteristics were well balanced between the two arms (Table 1). The median age was 85 years [interquartile range (IQR), $81-88$ years], and $43.1 \%$ of the participants were male. The Barthel index of activities of daily living (ADLs) as modified by Collins and Wade, scores the Barthel Index in one-point increments, ranging 0-20 and indicates the patients function prior to admission [30]. Barthel Index was assessed in 51 of 59 participants (86.5\%) in the intervention arm and 54 of 64 participants (84.4\%) in the control arm. The median Barthel index was 15.5 (IQR 10.8-20.0), which was similar between both groups and would indicate moderate functional impairment in both groups. Vaccination rates were low on admission [influenza vaccine, $4.9 \%$; 23 -valent pneumococcal polysaccharide vaccine (PPSV23), 0.8\%; 13-valent pneumococcal conjugate vaccine (PCV13), 5.7\%], and were not significantly different between the two arms.

\section{Primary and secondary outcomes}

There was no significant difference in the incidence of hospital-acquired pneumonia during hospitalisation. During hospitalisation, pneumonia was diagnosed in one of $59(1.7 \%)$ participants who received the multi-component intervention and three of 64 (4.7\%) participants who received usual care. One participant in the usual care group was deemed a protocol deviation as respiratory symptoms were present

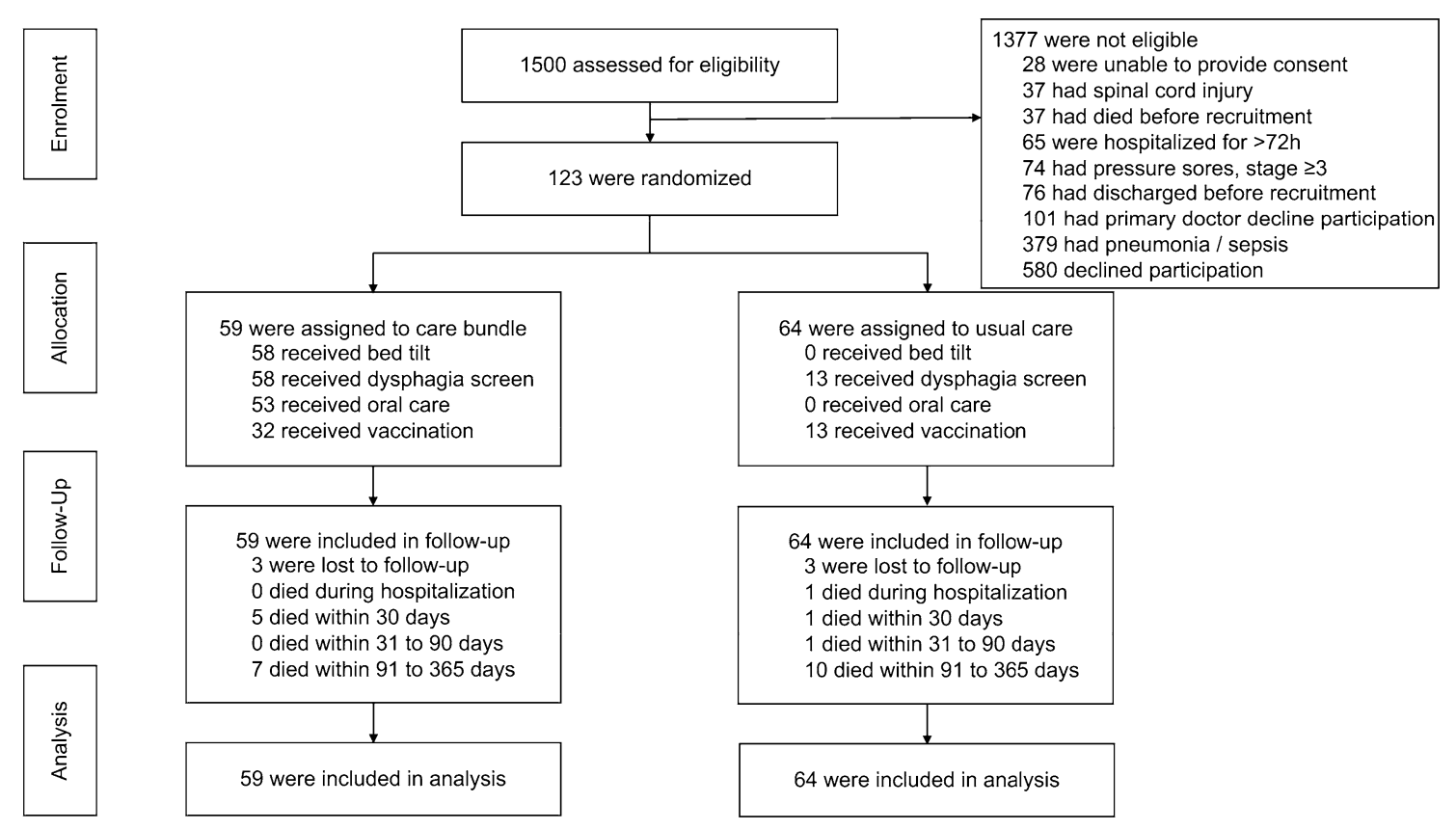

Fig. 2 Flow chart showing enrolment, randomization, allocation, follow-up and analysis phases 
Table 1 Baseline characteristics of study participants

\begin{tabular}{|c|c|c|c|c|}
\hline & All & Usual care (control) & Intervention & $P$ value \\
\hline Number of participants & 123 & 64 & 59 & \\
\hline Age, median (years) & 85 & 88 & 84 & 0.13 \\
\hline Interquartile range & $(81-88)$ & $(82-89)$ & $(81-88)$ & \\
\hline Min-max & $71-101$ & $71-97$ & $71-101$ & \\
\hline Male & $53(43.1 \%)$ & $24(37.5 \%)$ & $29(49.2 \%)$ & 0.19 \\
\hline Ethnicity & & & & 0.79 \\
\hline Chinese & $86(69.9 \%)$ & $46(71.8 \%)$ & $40(67.8 \%)$ & \\
\hline Malay & $26(21.2 \%)$ & $12(18.8 \%)$ & $14(23.7 \%)$ & \\
\hline Indian and others & $11(8.9 \%)$ & $6(9.4 \%)$ & $5(8.5 \%)$ & \\
\hline Barthel index & 15.5 & 15.5 & 15 & 0.71 \\
\hline Interquartile range & $(10.8,20.0)$ & $(11,19.3)$ & $(10.0,20.0)$ & \\
\hline Number of participants & 106 & 54 & 51 & \\
\hline \multicolumn{5}{|l|}{ Vaccinations at baseline } \\
\hline Influenza & $6(4.9 \%)$ & $4(6.3 \%)$ & $2(3.4 \%)$ & 0.68 \\
\hline Pneumococcal PPSV23 & $1(0.8 \%)$ & $0(0.0 \%)$ & $1(1.7 \%)$ & 0.48 \\
\hline Pneumococcal PCV13 & $7(5.7 \%)$ & $4(6.3 \%)$ & $3(5.1 \%)$ & 1.00 \\
\hline \multicolumn{5}{|c|}{ Grouped discharge diagnoses } \\
\hline Falls related & $45(36.6 \%)$ & $26(40.6 \%)$ & $19(32.2 \%)$ & \\
\hline Cardiac & $17(13.8 \%)$ & $7(10.9 \%)$ & $10(16.9 \%)$ & \\
\hline Gastro-intestinal & $8(6.5 \%)$ & $2(3.1 \%)$ & $6(10.2 \%)$ & \\
\hline Infective episode & $13(10.6 \%)$ & $6(9.4 \%)$ & $7(11.8)$ & \\
\hline Musculoskeletal & $6(4.9 \%)$ & $4(6.2 \%)$ & $2(3.4 \%)$ & \\
\hline Neurological & $20(16.3 \%)$ & $12(18.7 \%)$ & $8(13.6 \%)$ & \\
\hline Respiratory & $1(0.8 \%)$ & $1(1.6 \%)$ & $0(0 \%)$ & \\
\hline Others & $13(10.6 \%)$ & $6(9.4 \%)$ & $7(11.8 \%)$ & \\
\hline Concomitant dementia & $7(5.7 \%)$ & $6(9.4 \%)$ & $1(2 \%)$ & \\
\hline
\end{tabular}

PPSV23 23-valent pneumococcal polysaccharide vaccine, PCV13 13-valent pneumococcal conjugate vaccine on admission. No significant difference was found in the proportions of patients who contracted pneumonia during hospitalisation when comparing the two groups (1.7\% vs. $4.7 \%, P=0.620$ ).

By contrast, the mean time from randomization to the next hospitalisation was significantly longer in the intervention group compared to the control group (11.5 months vs. 9.5 months; $P=0.049$; Fig. 3). One year after randomization, participants in the intervention group had a reduction in the risk of hospitalisation due to respiratory infections, compared to the control group (18.6\% vs. $34.4 \% ; P=0.049)$. The absolute risk reduction was $15.7 \%$ (95\% CI $0.43-31.03 \%$ ), and the number needed to treat to prevent one hospitalisation was 6.4 (95\% CI 3.2-233.3).

There was no significant difference in overall mortality between the intervention and control groups $(20.3 \%$ vs $20.3 \% ; P=1.00 ; P=0.99$ ) (Table 2). Two patients in the intervention group transferred to the palliative care unit following their index admission due to diagnoses identified during their hospital admission. There was no significant difference in respiratory-related mortality between the intervention and control groups (5.1\% vs $10.9 \% ; P=0.34)$. There was no difference in the non-respiratory-related mortality between the intervention and control groups $(15.25 \%$ vs $9.4 \% ; P=0.23$ ).

The multi-component intervention, which included routine dysphagia screening, increased the frequency of both screening and diagnosis of oropharyngeal dysphagia (Table 3). The number of participants screened for oropharyngeal dysphagia increased by $74.9 \%$. As a result, the diagnosis of oropharyngeal dysphagia increased by $15.3 \%$. Dysphagia screening was performed on 58 participants (98.3\%) in the intervention arm and 15 participants (23.4\%) in the control arm. Subsequently, oropharyngeal dysphagia was diagnosed more frequently in the intervention arm. Oropharyngeal dysphagia was diagnosed in 21 participants (35.6\%) in the intervention arm and 13 participants (20.3\%) in the control arm $(35.6 \%$ vs. $20.3 \%$; $P=0.001)$.

Vaccination rates improved by offering appropriate vaccinations on discharge (Table 3 ). The vaccination rate was significantly higher in the intervention group for both PCV13 and influenza vaccinations. PCV13 vaccination 
Fig. 3 Proportion of participants with re-admissions to hospital for acute respiratory infections over time. Log-rank test comparing the prevention bundle to usual care shows a significant difference in mean time from randomization to next hospitalization due to acute respiratory infections $(9.5$ months vs. 11.5 months; $P=0.049$ ). One year after randomization, the intervention group had a lower risk of hospitalization for acute respiratory infections (18.6\% vs. $34.4 \% ; P=0.049)$
Table 2 Study outcomes from multi-component interventions

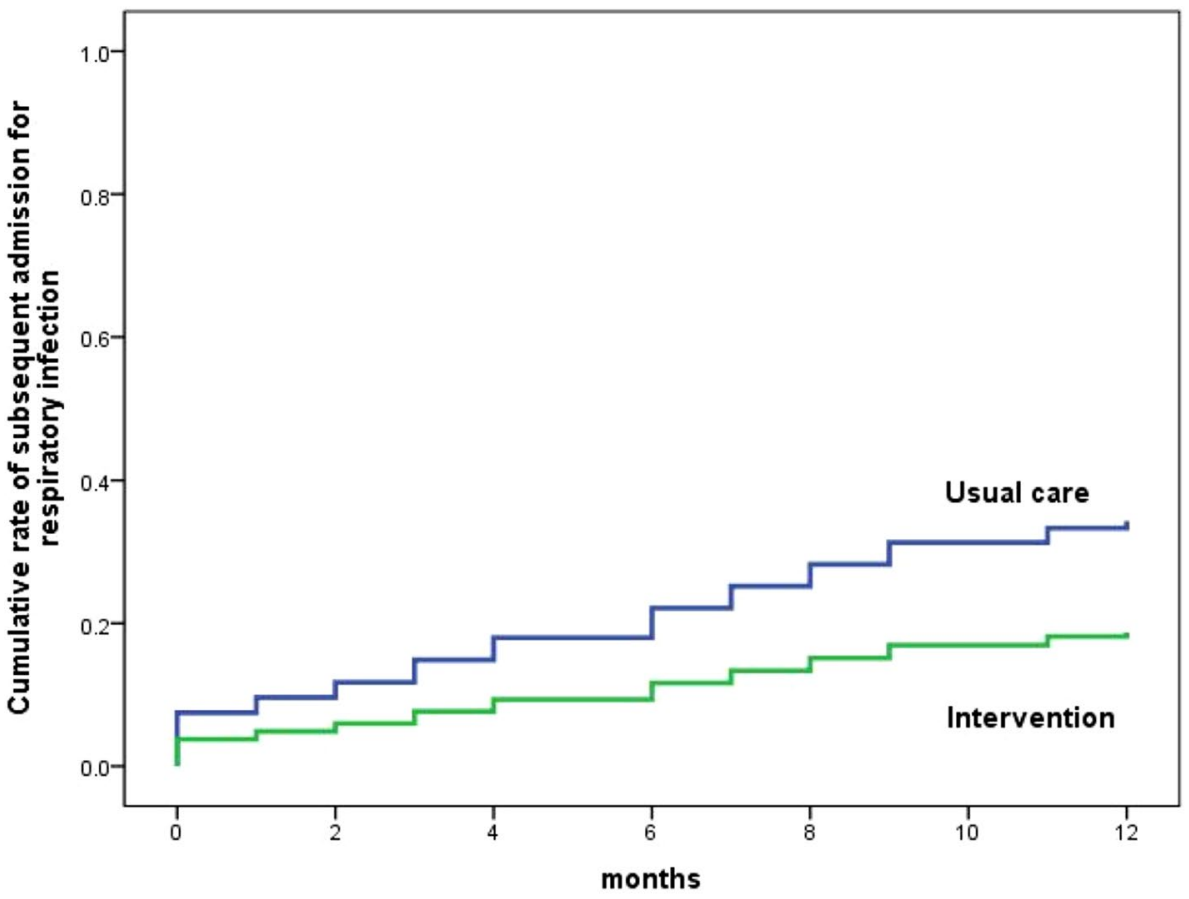

\begin{tabular}{|c|c|c|c|c|}
\hline Study outcomes & All & Usual care & Intervention & $P$ value \\
\hline \multicolumn{5}{|l|}{ Hospital length of stay } \\
\hline Number of participants & 123 & 64 & 59 & \multirow{3}{*}{0.86} \\
\hline Median hospital LOS (days) & 8 & 8 & 8 & \\
\hline Interquartile range & $(5-12)$ & $(5-11)$ & $(5-13)$ & \\
\hline \multicolumn{5}{|l|}{ 30-day hospital re-admission } \\
\hline Number of participants & 122 & 64 & 58 & \\
\hline No & $88(72.1 \%)$ & $46(71.9 \%)$ & $42(72.4 \%)$ & \multirow[t]{2}{*}{0.95} \\
\hline Yes & $34(27.9 \%)$ & $18(28.1 \%)$ & $16(27.6 \%)$ & \\
\hline \multicolumn{5}{|l|}{ 30-day respiratory re-admission } \\
\hline Number of participants & 34 & 18 & 16 & \multirow[t]{3}{*}{0.34} \\
\hline No & $29(85.3 \%)$ & $14(77.8 \%)$ & $15(93.8 \%)$ & \\
\hline Yes & $5(14.7 \%)$ & $4(22.2 \%)$ & $1(6.3 \%)$ & \\
\hline \multicolumn{5}{|l|}{ Mortality overall $n(\%)$} \\
\hline No & & $51(79.7 \%)$ & $47(79.7 \%)$ & \multirow[t]{5}{*}{0.99} \\
\hline Yes & & $13(20.3 \%)$ & $12(20.3 \%)$ & \\
\hline$<30$ days of discharge & & $2(3.1 \%)$ & $5(8.4 \%)$ & \\
\hline 30-90 days of discharge & & $1(1.6 \%)$ & $0(0.0 \%)$ & \\
\hline 90 days -1 year of discharge & & $10(15.6 \%)$ & $7(11.9 \%)$ & \\
\hline \multicolumn{5}{|l|}{ Cause of mortality } \\
\hline Non-respiratory related & & $6(9.4 \%)$ & $9(15.2 \%)$ & \\
\hline Respiratory infection & & $7(10.9 \%)$ & $3(5.1 \%)$ & 0.34 \\
\hline
\end{tabular}

was given to 32 participants $(50.8 \%)$ in the intervention group and 13 patients $(20.3 \%)$ in the control group $(50.8 \%$ vs. $20.3 \% ; P<0.001)$. Influenza vaccination was received in 32 participants $(54.5 \%)$ in the intervention group and 11 $(17.2 \%)$ in the control group $(54.5 \%$ vs. $17.2 \% ; P<0.001)$.
Those that did not receive vaccination were predominantly due to patient or family declining vaccination. PPSV23 vaccination was administered to one patient $(1.7 \%)$ in the intervention group and none in the control group $(1.7 \%$ vs. $0.0 \% ; P=0.48)$. The majority of vaccination in the 
Table 3 Implementation of prevention bundle components

\begin{tabular}{|c|c|c|c|c|}
\hline Bundle component & All & Usual care & Intervention & $P$ value \\
\hline Number of participants & 123 & 64 & 59 & \\
\hline \multicolumn{5}{|l|}{ Dysphagia } \\
\hline Not screened & $50(40.7 \%)$ & $49(76.6 \%)$ & $1(1.7 \%)$ & $<0.001$ \\
\hline Screened & $73(59.3 \%)$ & $15(23.4 \%)$ & $58(98.3 \%)$ & \\
\hline No dysphagia & $39(31.7 \%)$ & $2(3.1 \%)$ & $37(62.7 \%)$ & \\
\hline Present & $34(27.6 \%)$ & $13(20.3 \%)$ & $21(35.6 \%)$ & \\
\hline Cognitive dysphagia & 2 & 1 & 1 & \\
\hline Mild dysphagia & 21 & 8 & 13 & \\
\hline Mild-to-moderate & 6 & 2 & 4 & \\
\hline Moderate & 1 & 0 & 1 & \\
\hline Presbyphagia & 4 & 2 & 2 & \\
\hline Oral hygiene & $53(43.1 \%)$ & $0(0.0 \%)$ & $53(89.8 \%)$ & \\
\hline Adherence to mouthwash & & & $23(38.9 \%)$ & \\
\hline Bed tilt & $58(47.2 \%)$ & $0(0.0 \%)$ & $58(98.3 \%)$ & \\
\hline \multicolumn{5}{|l|}{ Vaccinations during study } \\
\hline Influenza & $42(34.1 \%)$ & $11(17.2)$ & $32(54.5 \%)$ & $<0.001$ \\
\hline \multicolumn{5}{|l|}{ Pneumococcal } \\
\hline PPSV23 & $1(0.8 \%)$ & $0(0.0 \%)$ & $1(1.7 \%)$ & 0.48 \\
\hline PCV13 & $43(35.0 \%)$ & $13(20.3 \%)$ & $30(50.8 \%)$ & $<0.001$ \\
\hline
\end{tabular}

PPSV23 23-valent pneumococcal polysaccharide vaccine, PCV13 13-valent pneumococcal conjugate vaccine

intervention group took place before discharge from hospital, however due to clinical reasons such as sepsis, some patients were vaccinated after discharge. Following discharge, seven patients in the intervention group received both influenza and pneumococcal vaccination, with mean time to vaccination of 28.7 days, median 29 days and range of 1-71 days. Patients in the usual care group could request vaccination but it was not offered routinely and no patients were vaccinated during their hospital stay but 9 patients received influenza and pneumococcal vaccination post discharge, with an average time to vaccination of 207.7 days, median 203 days and range 98-333 days. Of these nine patients, seven received both vaccinations at one visit and two received their vaccinations 3 months apart.

Adherence to regular oral care, defined as cleaning the mouth at least twice a day, was improved by reducing the frequency of serving chlorhexidine oral care from four to two times a day. Adherence rates improved from $16.1 \%$ (6 of 35 participants) to $94 \%$ (17 of 18 participants) when the frequency of chlorhexidine gargle was reduced on August 1,2017 . Overall adherence was $38.9 \%$ ( 23 of 59 participants). Conversely, adherence to whole bed tilt (Fig. 3) was high at $98.3 \%$ (58 of 59 participants). One patient declined whole bed tilt due to discomfort.

\section{Discussion}

To our knowledge, this randomized, parallel-group, controlled trial is the first study that aims to evaluate a multicomponent intervention, including reverse Trendelenburg position, for hospital-acquired pneumonia prevention in older hospitalized patients. While our data did not show that the intervention prevented pneumonia during hospitalisation, it did demonstrate that a multi-component intervention can prevent future hospitalisations for respiratory infections. Prevention of future hospitalisations reduces the risk of future nosocomial infections, increases disability-free life expectancies, lowers healthcare costs, alleviates the burden on healthcare resources and reduces patients' frequency of respiratory symptoms. In addition, the multi-component intervention significantly increased the frequency of diagnosis of oropharyngeal dysphagia and improved vaccination rates, which prevents future respiratory infections and enhances herd immunity.

We postulate that the prevention of subsequent hospitalisations for respiratory infections was achieved for a number of reasons. First, prompt diagnosis of oropharyngeal dysphagia ensures patients are served the appropriate dietary consistency, thereby reducing the risk of aspiration during meals and after discharge. One quarter of hospital acquired pneumonia is due to aspiration [28] and older patients with swallowing problems are at greater risk for aspiration pneumonia, particularly those with cognitive impairment $[29$, 
31]. In one study, over $52.6 \%$ patients admitted to a geriatric unit with an acute medical illness had oropharyngeal dysphagia [32] and 55\% of patients presenting with pneumonia had clinical signs of oropharyngeal dysphagia [33]. Despite its prevalence and severity, oropharyngeal dysphagia, a potentially modifiable risk to older patients, is still underdiagnosed and untreated in many medical centers [33]. The European Society for Swallowing Disorders proposed that dysphagia screening should be included in all standard screening protocols, to implement treatment strategies and prevent its main complications [34]. A conceivable future strategy would be to screen all patients over the age of 80 for dysphagia during their acute hospital admission by introducing a simple swallow screen (Fig. 4) administered by nursing staff.

Second, oral care with chlorhexidine, diminishes colonization of the oral cavity and gastrointestinal tract by multi-drug resistant organisms during hospitalisation. Oral cleansing in itself is beneficial as it reduces pneumonia in both edentulous and dentate subjects, which suggests that oral colonization of bacteria contributes to hospital acquired pneumonia to a greater extent than periodontitis [35]. Poor
Fig. 4 Dysphagia screen undertaken by Research Team Members (adapted from the Toronto Bedside Swallowing Screening Test (TOR-BSST) (Martino et al., 2008))

\section{Dysphagia Screening Form for HAP Study}

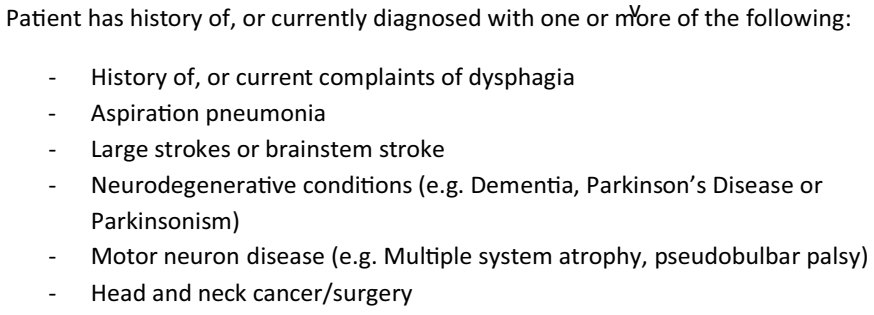

- Unable to maintain alertness for at least 10 minutes while sitting upright

- SOB requiring oxygen supplementation via face mask

$$
\text { If all 'no' }
$$

Do not proceed with If one or screening. more 'yes' ST to assess

Give 3 teaspoons of water to patient. Feed slowly and carefully. Are any of the following signs present?

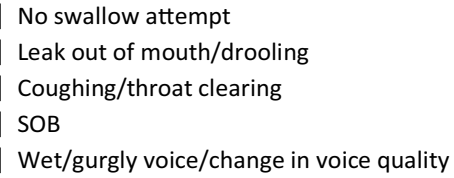

$\begin{array}{cl} & \text { Do not } \\ & \text { proceed } \\ & \text { with } \\ \text { If one or } & \text { screening. } \\ \text { more 'yes' } & \text { ST to assess }\end{array}$

Stop feeding. Patient fails dysphagia screening. ST to assess

Stop feeding. Patient fails dysphagia If one or screening. more 'yes' ST to assess

\section{Patient passes dysphagia screening.}


periodontal care, the presence of biofilm and reduced mechanical cleansing are shown to contribute to aspiration pneumonia [35-37] and oral care is a modifiable factor, easily enhanced in a ward setting [38]. Patient compliance to oral care was improved in this study with a twice daily prescribed oral care regimen.

Third, the routine vaccinations boost participants' immunity to influenza and pneumococcus after discharge. Older people with influenza infections have the highest risk of death, highest risk of hospitalisation and the lowest likelihood of admission to ICU compared to younger people [39]. Locally the influenza-associated hospitalisation rate is $16.3 \%$, with a substantially increased excess hospitalisation rates in those age 75 years and above [20] but community uptake of these vaccines is poor as evidenced by the very low pre-study vaccination rates in this aged cohort, $4.9 \%$ for prior influenza and $6.5 \%$ for prior pneumococcal vaccination $[21,40]$. The World Health Organisation recommends countries aim achieve influenza vaccination coverage of $75 \%$, but there are major differences across European countries and historically poor uptake in older people [41]. The vaccination strategy in this study was opportunistic and likely still beneficial as there are two seasonal peaks of influenza in Singapore, which correspond to the Northern and Southern Hemisphere winter seasons. Whether this strategy could be beneficial in other European countries would need to further investigation. There were no adverse events reported and no increase in 30-day re-admissions using an opportunistic vaccination strategy. The numbers of patients who were vaccinated against both influenza $(54.5 \%$ vs. $17.2 \%$; $P<0.001)$ and pneumococcus $(52.5 \%$ vs. $20.3 \% ; P<0.001)$ increased significantly in the intervention group, which may have helped to reduce respiratory re-admissions. A potential confounding factor could be the increased uptake of vaccination in the control group, although the late vaccination of these patients makes this less likely. The study may have impacted the patient's belief system to improve vaccination uptake as some patients did not feel vaccinations were beneficial. Cost was less of an influencing factor, as vaccinations are subsidized but chargeable locally, and despite vaccinations being offered at no cost to patients in the intervention group, vaccination uptake was just over $54.5 \%$ for influenza and $52.5 \%$ for pneumococcal vaccine.

Reverse Trendelenburg is a novel aspect of this study, and was well tolerated by patients. Patient positioning during hospitalisation is gaining momentum as a preventative strategy, with twice-daily elevation of the head of the bed shown to reduce pneumonia by $26 \%$ outside of an ICU setting [42]. The majority of studies and guidelines aimed at preventing hospital acquired pneumonia have utilized semi-recumbent positioning of $30^{\circ}-45^{\circ}[4,19,42-44]$ but it is conceivable that intra-abdominal pressure and gastro-oesphageal aspiration would be reduced using reverse Trendelenburg positioning compared to elevation of the head of the bed.

This study has its limitations. First, due to slow recruitment, the study was terminated before reaching the calculated sample size. As a result, there were only four pneumonias recorded among our participants during hospitalisation, making the study underpowered for its first primary outcome measure. Future studies may need a larger sample size, as disparity between suspected diagnosis of hospital acquired pneumonia (10.9\%) and those meeting-defined diagnostic criteria (5.8\%) is a recognized limitation of studies [29].

Second, due to the visibly different care received by the two arms, it was difficult to maintain blinding among the healthcare and study teams and patient outcomes were not blinded which may introduce bias into the study. Third, since this study was only undertaken at one hospital, it is unclear if the multi-component intervention can be replicated at other settings. Fourth, the tight time frame for recruitment, with $72 \mathrm{~h}$ from hospital admission made recruitment challenging. Recruitment was particularly difficult to achieve for those that needed LAR consent within $72 \mathrm{~h}$ of admission. The most frequent reason cited for declining the study being "too old to participate in a research study". Widening the time window for recruitment would improve recruitment, but would increase the likelihood of recruiting a patient with existing infection. Fifth, information was collected on primary discharge diagnoses but not co-morbidities and this may influence the study findings, as there were fewer primary diagnoses of dementia in the intervention group.

Moving forward, future studies are needed to confirm and clarify our results. For instance, trials at different healthcare institutions would determine if our multi-component intervention may reduce recurrent hospitalisations at other settings, such as subacute hospitals and nursing homes. Trials that evaluate individual components of the care bundle may identify the parts of the care bundle which are most effective, or even essential. Future studies that stratify participants based on frailty scores or respiratory comorbidities (such as asthma, chronic obstructive pulmonary disease, lung cancer or frailty) may also determine whether these groups would benefit more from the multi-component interventions.

This study shows that a multi-component intervention for nosocomial pneumonia can prevent future hospitalisations for respiratory infections in older adults, but may not significantly reduce the incidence of hospital-acquired pneumonia during hospitalisation. Our data suggests that interventions implemented during hospitalisation may improve the long-term well-being of older patients long after discharge. Therefore, clinicians should consider how they can proactively prevent repeat hospitalisations by taking preventive measures during the hospital stay itself. During the COVID-19 pandemic, hopefully, the 
multi-component interventions described will inspire clinicians to envision new ways to make hospitalisation a safer experience for older adults.

Acknowledgements We thank Ms. Tao Chunfeng and Ms. Geraldine Lim from the Research Department at Changi General Hospital.

Author contributions Designed the study: BHR, HS, and AAMT. Implemented the trial: BHR, HS, AS, GKP, WSTA, YQL, LFZ, YLZ, RBS and AAMT. Statistical analysis: ACAY, LYT and PTT. Drafted the report: BHR, LYT and ACAY. All authors critically reviewed the report for important intellectual content and approved the final submitted version.

Funding The study was supported by a Changi General Hospital Research Grant. Grant number CHF2016.04-P. The funding agency played no role in the design, conduct, analysis and the interpretation of the data, or the writing of the report.

\section{Declarations}

Conflict of interest The main author attended a Pneumococcal Vaccine Expert Input forum in 2019 (an honorarium was received and donated to the Geriatric Department) and received sponsorship from Pfizer to attend an Asia Pneumococcal and Meningococcal Disease Conference in Hong Kong in 2016. The remaining authors declare no conflict of interest, and this paper has not been published or presented elsewhere.

Ethical approval The trial was conducted in accordance with the Declaration of Helsinki, and the study protocol was approved by the SingHealth Centralized Institutional Review Board.

Informed consent Written informed consent was obtained by a study team member and if the patient lacked capacity to make decisions then consent was obtained from a legally appointed representative (LAR).

Impact statement We certify that this work is novel. It contributes to the literature because, to our knowledge, it is the first randomized, controlled trial that evaluates the effectiveness of a multicomponent interventions, including reverse Trendelenburg position, designed to prevent nosocomial pneumonia in older adults admitted to the general ward.

\section{References}

1. Guidelines for the management of adults with hospital-acquired, ventilator-associated, and healthcare-associated pneumonia. Am J Respir Crit Care Med. 2005;171:388-416. https://doi.org/10. 1164/rccm.200405-644ST

2. Magill SS, O'Leary E, Janelle SJ, Thompson DL, Dumyati G, Nadle J et al (2018) Changes in prevalence of Health Care-Associated Infections in US Hospitals. N Engl J Med 379(18):17321744. https://doi.org/10.1056/NEJMoa1801550

3. Kalil AC, Metersky ML, Klompas M, Muscedere J, Sweeney DA, Palmer LB et al (2016) Management of adults with hospitalacquired and ventilator-associated pneumonia: 2016 Clinical Practice Guidelines by the Infectious Diseases Society of America and the American Thoracic Society. Clin Infect Dis 63:e61-e111

4. Alexiou VG, Ierodiakonou V, Dimopoulos G, Falagas ME (2009) Impact of patient position on the incidence of ventilator-associated pneumonia: a meta-analysis of randomized controlled trials. J Crit Care 24:515-522

5. Hua F, Xie H, Worthington HV, Furness S, Zhang Q, Li C (2016) Oral hygiene care for critically ill patients to prevent ventilatorassociated pneumonia. Cochrane Database Syst Rev. https://doi. org/10.1002/14651858.CD008367.pub3

6. Klompas M, Branson R, Eichenwald EC, Greene LR, Howell $\mathrm{MD}$, Lee G et al (2014) Strategies to prevent ventilator-associated pneumonia in Acute Care Hospitals: 2014 update. Infect Control Hosp Epidemiol 35(8):915-936

7. Wip C, Napolitano L (2009) Bundles to prevent ventilator-associated pneumonia: how valuable are they? Curr Opin Infect Dis 22(2): 159-166

8. Torres A, Niederman MS, Chastre J, Ewig S, Fernandez-Vandellos P, Hanberger $\mathrm{H}$ et al (2017) Task force report ERS/ESICM/ ESCMID/ALAT guidelines. Eur Respir J 50:1700582. http:// ow.ly/dGhv30dAVoa

9. Torres A, Ewig S, Lode H, Carlet J (2009) Defining, treating and preventing hospital acquired pneumonia: European perspective. Intensive Care Med 35:9-29

10. Teramoto S, Yoshida K, Hizawa N (2015) Update on the pathogenesis and management of pneumonia in the elderly-roles of aspiration pneumonia. Respir Investig 53:178-184

11. Marik P, Kaplan D (2003) Aspiration pneumonia and dysphagia in the elderly. Chest 124:328-336

12. Clavé P, Rofes L, Arreola V, Almirall J, Cabré M, Campins L et al (2011) Diagnosis and management of oropharyngeal dysphagia and its nutritional and respiratory complications in the elderly. Gastroenterol Res Pract. https://doi.org/10.1155/2011/ 818979

13. Turley R, Cohen S (2009) Impact of voice and swallowing problems in the elderly. Otolaryngol Head Neck Surg Off J Am Acad Otolaryngol Head Neck Surg 140(1):33-36. http://www.ncbi.nlm. nih.gov/pubmed/19130958

14. Rapp L, Sourdet S, Vellas B, Lacoste-Ferré M-H (2017) Oral health and the frail elderly. J Frailty Aging 6(3):154-160

15. Tanaka T, Takahashi K, Hirano H, Kikutani T, Watanabe Y, Ohara $Y$ et al (2018) Oral frailtyasa risk factor for physical frailty and mortality in community-dwelling elderly. J Gerontol Ser A Biol Scie Med Sci 73(12):1661-1667

16. Tesoro M, Peyser D, Villarente F (2018) A retrospective study of non-ventilator-associated hospital acquired pneumonia incidence and missed opportunities for nursing care. J Nurs Adm 48(5):285-291

17. Higashiguchi T, Ohara H, Kamakura Y, Kikutani T, Kuzuya M, Enoki $\mathrm{H}$ et al (2017) Efficacy of a new post-mouthwash intervention (wiping plus oral nutritional supplements) for preventing aspiration pneumonia in elderly people: a multicenter, randomized, comparative trial. Original Paper Ann Nutr Metab 71:253-60. www.karger.com/anm

18. Garrouste-Orgeas M, Chevret S, Arlet C, Marie O, Rouveau M, Popoff $\mathrm{N}$ et al (1997) Oropharyngeal or gastric colonization and nosocomial pneumonia in adult intensive care unit patients: a prospective study based on genomic DNA analysis. Am J Respir Crit Care Med 156(5):1647-1655. https://doi.org/10.1164/ajrccm. 156.5.96-04076

19. Safdar N, Crnich CJ, Maki DG (2005) The pathogenesis of ventilator-associated pneumonia: its relevance to developing effective strategies for prevention. Respir Care 50(6):725-739

20. Ng Y, Chua LAV, Ma S, Jian MLV (2019) Estimates of influenzaassociated hospitalisations in tropical Singapore, 2010-2017: higher burden estimated in more recent years. Influenza Other Respir Viruses 13(6):574-581

21. Ang LW, Cutter J, James L, Goh KT (2017) Factors associated with influenza vaccine uptake in older adults living in the community in Singapore. Epidemiol Infect 145(4):775-786 
22. Ho HJ, Tan Y-R, Cook AR, Koh G, Yean Tham T, Anwar E et al (2019) Increasing influenza and pneumococcal vaccination uptake in seniors using point-of-care informational interventions in primary care in Singapore: a pragmatic, cluster-randomized crossover trial. Am J Public Health 109:1776-83. http://www.ajph.org

23. Matanock A, Lee G, Gierke R, Kobayashi M, Leidner A, Pilishvili $\mathrm{T}$ (2019) Use of 13-valent pneumococcal conjugate vaccine and 23 -valent pneumococcal polysaccharide vaccine among adults aged $\geq 65$ years: updated recommendations of the advisory committee on immunization practices. MMWR Morb Mortal Wkly Rep 68(46):1069-1075. http://www.cdc.gov/mmwr/volumes/68/ $\mathrm{wr} / \mathrm{mm} 6846 \mathrm{a} 5 . \mathrm{htm}$ ?s_cid=mm6846a5_w

24. Dunham CM, Hileman BM, Hutchinson AE, Antonaccio T, Chance EA, Huang GS et al (2017) Evaluation of operating roomreverse Trendelenburg positioning and its effect on postoperative hypoxemia, aspiration, and length of stay: a retrospective studyof consecutive patients. Perioper Med 6(1):1-11

25. Martino R, Silver F, Teasell R, Bayley M, Nicholson G, Streiner DL et al (2009) The toronto bedside swallowing screening test (TOR-BSST) development and validation of a dysphagia screening tool for patients with stroke. Stroke 40(2):555-561

26. Zand F, Zahed L, Mansouri P, Dehghanrad F, Bahrani M, Ghorbani M (2017) The effects of oral rinse with $0.2 \%$ and $2 \%$ chlorhexidine on oropharyngeal colonization and ventilator associated pneumonia in adults' intensive care units. J Crit Care 40:318-322. https://doi.org/10.1016/j.jcrc.2017.02.029

27. Özçaka Ö, Başoğlu ÖK, Buduneli N, Taşbakan MS, Bacakoğlu F, Kinane DF (2012) Chlorhexidine decreases the risk of ventilator-associated pneumonia in intensive care unit patients: a randomized clinical trial. J Periodontal Res 47(5):584-592

28. See I, Chang J, Gualandi N, Buser GL, Rohrbach P, Smeltz DA et al (2016) Clinical correlates of surveillance events detected by National Healthcare Safety Network Pneumonia and Lower Respiratory Infection Definitions-Pennsylvania, 2011-2012. Infect Control Hosp Epidemiol 37(7):818-824. https://www.cambridge. org/core/product/identifier/S0899823X1600074X/type/journal_ article

29. Burton LA, Price R, Barr KE, Mcauley SM, Allen JB, Clinton AM et al (2016) Hospital-acquired pneumonia incidence and diagnosis in older patients. Age Ageing 45(1):171-174

30. Collin C, Wade DT, Davies S, Horne V (1988) The barthel ADL index: a reliability study. Disabil Rehabil 10(2):61-63. https://doi. org/10.3109/09638288809164103

31. Naruishi K, Nishikawa Y, Kido J, Fukunaga A, Nagata T (2018) Relationship of aspiration pneumonia to cognitive impairment and oral condition: a cross-sectional study. Clin Oral Investig 22(7):2575-2580

32. Carrión S, Cabré M, Monteis R, Roca M, Palomera E, Clavé P (2012) OP018 association between oropharyngeal dysphagia and malnutrition in elderly patients with acute diseases admitted to a general hospital. Clin Nutr Suppl 7(1):8

33. Unit AG, de Cirera C, Unit IC, Service ID, de Bellvitge H (2010) Prevalence and prognostic implications of dysphagia in elderly patients with pneumonia. Age Ageing 39:39-45
34. Baijens LWJ, Clavé P, Cras P, Ekberg O, Forster A, Gerald F et al (2016) European Society for Swallowing DisordersEuropean Union Geriatric Medicine Society white paper: oropharyngeal dysphagia as a geriatric syndrome. Clin Interv Aging 11:1403-1428

35. Scannapieco FA, Shay K (2014) Oral health disparities in older adults: oral bacteria, inflammation, and aspiration pneumonia. Dent Clin North Am 58(4):771-782. https://doi.org/10.1016/j. cden.2014.06.005

36. Müller F (2015) Oral hygiene reduces the mortality from aspiration pneumonia in frail elders. J Dent Res 94(March):14S-16S

37. van der Maarel-Wierink CD, Vanobbergen JNO, Bronkhorst EM, Schols JMGA, de Baat C (2011) Risk factors for aspiration pneumonia in frail older people: a systematic literature review. $\mathrm{J}$ Am Med Direct Assoc. 12(5):344-354. https://doi.org/10.1016/j. jamda.2010.12.099

38. Baker D, Quinn B, Ewan V, Giuliano KK (2019) Sustaining quality improvement: long-term reduction of nonventilator hospitalacquired pneumonia. J Nurs Care Qual 34(3):223-229

39. Mertz D, Kim TH, Johnstone J, Lam PP, Science M, Kuster SP et al (2013) Populations at risk for severe or complicated influenza illness: systematic review and meta-analysis. BMJ (Online). 347(7923):1-15. https://doi.org/10.1136/bmj.f5061

40. Teo LM, Smith HE, Lwin MO, Tang WE (2019) Attitudes and perception of influenza vaccines among older people in Singapore: a qualitative study. Vaccine 37(44):6665-6672. https://doi.org/10. 1016/j.vaccine.2019.09.037

41. Jorgensen P, Mereckiene J, Cotter S, Johansen K, Tsolova S, Brown C (2018) How close are countries of the WHO European Region to achieving the goal of vaccinating $75 \%$ of key risk groups against influenza? Results from national surveys on seasonal influenza vaccination programmes, 2008/2009 to 2014/2015. Vaccine 36(4):442-452. https://doi.org/10.1016/j. vaccine.2017.12.019

42. Patty CM, Sandidge-Renteria A, Orique S, Dixon C, Camarena E, Newsom R et al (2021) Incidence and predictors of nonventilator hospital-acquired pneumonia in a community hospital. J Nurs Care Qual 36(1):74-78

43. Baker D, Quinn B (2018) Hospital acquired pneumonia prevention initiative-2: incidence of nonventilator hospital-acquired pneumonia in the United States. Am J Infect Control 46(1):2-7. https:// doi.org/10.1016/j.ajic.2017.08.036

44. Masterton RG, Galloway A, French G, Street M, Armstrong J, Brown E et al (2008) Guidelines for the management of hospitalacquired pneumonia in the UK: report of the working party on hospital-acquired pneumonia of the british society for antimicrobial chemotherapy. J Antimicrob Chemother 62(1):5-34

Publisher's Note Springer Nature remains neutral with regard to jurisdictional claims in published maps and institutional affiliations. 